American Journal of Infectious Diseases 4 (3): 187-192, 2008

ISSN 1553-6203

(C) 2008 Science Publications

\title{
Features in Tubercular Meningoencephalitis Diagnosis: 18 Childhood Cases
}

\author{
${ }^{1}$ P. Di Carlo, ${ }^{2}$ D. Cabibi, ${ }^{3}$ A. Casuccio, ${ }^{4}$ A. Mazzola, ${ }^{4}$ A. Romano, ${ }^{1}$ L. Titone \\ ${ }^{1}$ Istituto di Patologia Infettiva e Virologia, University of Palermo, Italy \\ ${ }^{2}$ Dipartimento di Patologia Umana, University of Palermo, Italy \\ ${ }^{3}$ Dipartimento di Neurologia, Oftalmologia, Otorinolaringoiatria e Psichiatria (Di.N.O.O.P.), \\ Sezione di Oftalmologia, University of Palermo, Italy \\ ${ }^{4}$ ARNAS Civico Benfratelli, G. Di Cristina and M. Ascoli, Palermo, Italy
}

\begin{abstract}
The aim of this research is to illustrate clinical and instrumental features of central nervous system tuberculosis (CNS-TB) in childhood in order to allow prompt diagnosis and adequate patient management. TB remains one of the most important communicable diseases and represents a major global health problem. Although pulmonary TB tends to be the most common form of the disease, the highest mortality and morbidity occurs with TB of the central nervous system (CNS-TB), which develops in $4 \%$ of children with tuberculosis. It has a high fatality rate and causes serious sequelae, especially during childhood. CT and MR imaging studies of 18 patients (11 female, 7 male, mean age 45.72 months) were retrospectively reviewed. A basilar enhancement was detected in all the patients. Other findings were hydrocephalus $(61 \%)$, intracranial tuberculomas $(17 \%)$ other nodular $(11 \%)$ and ischemic lesions (11\%). Edema, either perilesional or diffuse, was found in 7/18 patients. Severe ventricular dilatation was observed in $9 / 18$ cases. Two patients with fatal outcome had severe hydrocephalus associated with extensive ischemic areas in one case and intraventricular haemorrhage in the other. Cross-sectional imaging, together with clinical data, may provide useful clues for the early diagnosis of CNS-TB and help to prevent unnecessary morbidity and mortality.
\end{abstract}

Key words: Tuberculous meningitis, children, imaging

\section{INTRODUCTION}

Tuberculosis (TB) remains one of the most important communicable diseases and represents a major global health problem. Nearly a third of the world's population is infected with Mycobacterium tuberculosis, 8 million people develop active disease and 3 million die of it each year ${ }^{[1]}$. In Italy, as in other parts of the developed world, there has been a resurgence of TB since the mid $1980 \mathrm{~s}^{[1,2]}$.

Although pulmonary TB tends to be the most common form of tuberculosis, the highest mortality and morbidity occurs with TB of the central nervous system (CNS-TB) which develops in $4 \%$ of children with tuberculosis. It has a high fatality rate and causes serious sequelae, especially during childhood. In pediatric patients, CNS-TB frequently results in mental retardation, hydrocephalus, paraplegia, cranial nerve paralysis and death ${ }^{[3,4]}$.

CNS-TB occurs either in a diffuse form as basal exudative leptomeningitis or in a localized form as tuberculomas, abscess or cerebritis ${ }^{[5]}$.
Diagnostic evaluation includes tuberculin skin tests, chest radiography and other imaging studies, smear culture, examination of cerebrospinal fluid and polymerase chain reaction, as well as more recent immunological assays such as interferon- $\gamma$ or $\gamma \delta \mathrm{T}$ cells $s^{[6,7]}$. Modern imaging is a cornerstone of early CNS-TB diagnosis and may help to prevent unnecessary morbidity and mortality. Imaging techniques have recently been revealed as important diagnostic tools as they can demonstrate various conditions, such as intracranial tuberculomas, edema, ventricular dilatation and basal cistern enhancement ${ }^{[8,9]}$.

Contrast-enhanced MR imaging is generally the preferred procedure to detect and assess CNS tuberculosis ${ }^{[10]}$.

As CNS-TB is often clinically indolent and early treatment is necessary to avoid a fatal outcome, our aim was to seek to improve the diagnosis of the disease. We reviewed features of CNS-TB in an attempt to facilitate early diagnosis and enable prompt and adequate treatment of this serious, paediatric intracranial infection.

Corresponding Author: Daniela Cabibi, Dipartimento di Patologia Umana, Università degli Studi, Palermo Via del Vespro, 129 Palermo, Italy Tel: +39 0916553533 Fax: +39 0916553549 


\section{MATERIALS AND METHODS}

Patient cohort: We reviewed the medical records of all children admitted to hospital with suspected CNS-TB between January 1994 and March 2005. Eighteen cases (mean age 45, 72 months, SD 40, 67) met the diagnostic criteria for CNS-TB.

Diagnosis was made according to the clinical and/or microbiological criteria included in the Tuberculosis Case Definition ${ }^{[11]}$.

Patients were classified according to Medical Research Council Staging ${ }^{[12]}$ : Stage I (early stage) indicated patients in a febrile state, with other nonspecific symptoms and no neurological signs, stage II, neurological signs without marked sensorial changes, stage III (advanced stage), severe neurological signs, sensorial changes and/or coma.

The baseline characteristics of the TB patients are reported in Table 1 . The sample consisted of children from the same geographical area and with a similar socio-economic background. None of the children had been vaccinated with BCG in infancy. None of the case or control patients had signs of human immunodeficiency virus (HIV) infection, or was being treated with steroids or anti-tubercular drugs at the time of diagnosis and first sampling.

All the patients were inserted in a 36-month follow-up programme. Patients were considered as having severe neurological sequelae if they were unable to lead an independent existence and/or required the equivalent of full-time nursing care.

Image acquisition: The neuroradiological investigations were performed during hospitalization, based on the patients' clinical status.
The reviewer was a paediatric radiologist with an interest in neuroradiology and extensive experience in TB imaging.

CT-Eleven patients underwent CT scanning, at $3 \mathrm{~mm}$ collimation with a $5 \mathrm{~mm}$ interval, of the posterior fossa and at $10 \mathrm{~mm}$ collimation and interval above the tentorium cerebelli. All the images were obtained after the intravenous administration of iodinate low-osmolar contrast medium.

MRI-Nine patients underwent MRI examinations performed with the following parameters: following the routine spin echo (SE) T1 weighted (TR: 400-700 msec/TE: $15-25 \mathrm{msec}$ ) and T2 weighted (TR: 2,0004,000 msec/TE: $90-100 \mathrm{msec}$ ) series in the transverse plane, paramagnetic contrast media were applied. In some cases, T2 and/or T1 weighted coronal sections were added to permit a better localization of the lesions. SE T1 weighted images were obtained in the transverse, coronal and sagittal planes.

Treatment: Antituberculous treatment was administered for one year and consisted of 2 months of isoniazid (INH), rifampicin (RMP), pyrazinamide (PZA) and streptomycin (SM) or ethambutol (ETB), followed by 10 months of RMP and $\mathrm{INH}^{[13]}$. Where necessary, corticosteroid therapy was added during the first 4-6 weeks. Compliance was assessed in interviews with parents. Some patients required a neurosurgical procedure, which mainly involved the insertion of a Rickham reservoir to measure and control CSF pressure and obtain further CSF samples for microbiological analysis. In those patients in whom severe hydrocephalus persisted, Rickham reservoirs were converted into a permanent ventriculoperitoneal (VP) shunt.

Table 1: Baseline characteristics of the CNS-TB patients

\begin{tabular}{|c|c|c|c|c|c|}
\hline $\mathrm{Pz}$ & Age & Days of illness before admission & Family history & PPD & M.tuberculosis identification from LCS \\
\hline 1 & 12 year & 10 days (Fever) & - & - & - \\
\hline 2 & 14 month & 20 days (Fever) & + & + & Ziehl Neelsen \\
\hline 3 & 10 year & 3 days (Fever, headache, dizziness) & + & + & Ziehl Neelsen \\
\hline 4 & 8 year & 10 days (Fever, headache, drowsiness) & - & - & Culture (Petragnani) \\
\hline 5 & 2 year & 13 days (Fever) & - & + & - \\
\hline 6 & 2 year & 15 days (Fever) & - & - & - \\
\hline 7 & 6 year & 7 days (Fever, headache, neck pain) & - & + & Culture (Petragnani) \\
\hline 8 & 6 year & 12 days (Fever, abdominal pain, vomiting) & - & + & - \\
\hline 9 & 5 year & 1 day (Fever, vomiting) & - & - & PCR \\
\hline 10 & 4 year & 9 days (Fever, vomiting, headache) & - & - & PCR \\
\hline 11 & 3 year & 5 days (Fever, vomiting) & - & - & Culture (BACTEC) \\
\hline 12 & 2 year & 3 days (Fever, vomiting) & - & - & - \\
\hline 13 & 10 month & 9 days (Fever) & + & - & Ziehl Neelsen PCR \\
\hline 14 & 16 month & 9 days (Fever) & + & - & - \\
\hline 15 & 10 month & 15 days (Fever) & - & - & PCR, Culture (BACTEC) \\
\hline 16 & 11 month & 45 days (Legs functional impairment) & - & - & - \\
\hline 17 & 3 year & 15 days (Weakness, abdominal pain) & - & + & Culture (BACTEC) \\
\hline 18 & 6 month & 7 days (Fever, growing weakness) & + & + & - \\
\hline
\end{tabular}


Am. J. Infect. Dis., 4 (3): 187-192, 2008

Table 2: Clinical and radiological features of CNS-TB in the children of our sample

\begin{tabular}{|c|c|c|c|c|c|c|}
\hline \multirow[b]{2}{*}{ Case } & \multirow[b]{2}{*}{ Age } & \multirow[b]{2}{*}{$\begin{array}{l}\text { Clinical } \\
\text { stage }\end{array}$} & \multicolumn{3}{|c|}{ Neuroimaging features in acute phase } & \multirow[b]{2}{*}{ Complication and/or Sequalae } \\
\hline & & & $\begin{array}{l}\text { Meningeal } \\
\text { enhancement }\end{array}$ & Hydrocephalus & Nodular lesion Ischemia-Infarct & \\
\hline 1 & 12 year & $\mathrm{I}$ & + & & & Nystagmus \\
\hline 2 & 14 month & II & + Basal cisterns & Lateral Ventricle & & \\
\hline 3 & 10 year & I & In meninges & & & \\
\hline 4 & 8 year & II & + Basal cisterns & & & \\
\hline 5 & 2 year & III & + & Tetraventricular & & \\
\hline 6 & 2 year & I & + Basal cisterns & Tetraventricular & & Ventricolo-peritoneal shunt \\
\hline 7 & 6 year & I & + & Tetraventricular & & \\
\hline 8 & 6 year & III & + & Tetraventricular & $\begin{array}{l}\text { Extensive ischemic } \\
\text { area in left internal } \\
\text { carotid district }\end{array}$ & Death \\
\hline 9 & 5 year & I & + Basal cisterns & & & \\
\hline 10 & 4 year & II & + & & & $\begin{array}{l}\text { Motor disorder, mental } \\
\text { retardation }\end{array}$ \\
\hline 11 & 3 year & II & + Basal cisterns & Tetraventricular & Nodular lesion & \\
\hline 12 & 2 year & I & + & Modeste & & \\
\hline 13 & 10 month & III & + Basal cisterns & $\begin{array}{l}\text { Tetraventricular with } \\
\text { extensive bleeding }\end{array}$ & & Death \\
\hline 14 & 16 month & III & + & & $\begin{array}{l}\text { Ischemic area in right } \\
\text { internal capsule }\end{array}$ & \\
\hline 15 & 10 month & II & + & $\begin{array}{l}\text { Tetraventricular and } \\
\text { periventricular interstitial } \\
\text { edema }\end{array}$ & & \\
\hline 16 & 11 month & II & + Basal cisterns & Triventricular & & $\begin{array}{l}\text { Ventricolo-peritoneal shunt, } \\
\text { motor disorder }\end{array}$ \\
\hline 17 & 3 year & I & + Basal cisterns & & $\begin{array}{l}\text { Single lesion in } \\
\text { right temporo-parietal } \\
\text { basal area }\end{array}$ & Neurosurgical intervention \\
\hline 18 & 6 month & III & + Basal cisterns & Tetraventricular & & $\begin{array}{l}\text { Ventriculo-peritoneal shunt, } \\
\text { ptosis and strabismus }\end{array}$ \\
\hline
\end{tabular}

$\mathrm{p}$ values were derived from 2-tailed ANOVA tests. $\mathrm{p}$ values $<0.05$ were considered significant.

Patients were subsequently followed monthly on a day hospital basis to monitor the efficacy of the treatment, moreover after discharge and until the end of treatment, periodic blood tests were carried out to identify possible side effects.

\section{RESULTS AND DISCUSSION}

Table 2 summarizes our results. On admission, 7 children were classified as having stage 1 disease, 6 with stage II and 5 with stage III, all the children aged < 2 years were in stage II or III.

All the patients had a meningeal enhancement, mostly involving the basal cistern. The imaging studies revealed hydrocephalus in 11 patients $(61 \%)$, nodular lesions in $2(11 \%)$ and ischemic areas in $2(11 \%)$.

Severe ventricular dilatation was observed in $9 / 11$ cases. In the patients with fatal outcome, severe hydrocephalus was associated with an extensive ischemic area in case $n^{\circ} 8$ (6 years of age) and with intraventricular haemorrhage in case $\mathrm{n}^{\circ} 13$ (10 months of age). Both patients died within ten days after hospitalization: case $n^{\circ} 13$ rapidly fell into a coma in the first 24 hours after admission.

Figure 1 and 2 show images of severe hydrocephalus.

Six children with hydrocephalus had recurrent seizures both on admission and during hospitalization. In cases 5 and 15, a Rickham reservoir was applied, leading to an improvement in their condition, whereas in cases 6,16 and 18 a ventricolo-peritoneal shunt was necessary.

Two children had nodular lesions: imaging showed signs of diffuse cerebritis in case 11 (Fig. 3) and a single growth suggestive of tuberculoma in case 17 (Fig. 4).

Both patients made a complete recovery: a prompt neurosurgical intervention was essential in case 17. Ischemic lesions were observed in two patients: a serious, extensive ischemic area in case 8 and a small ischemic area in the internal capsule in case 14 which did not prevent the patient from making a complete recovery.

Although ten children had clinical evidence of cranial nerve involvement on admission, MRI investigations revealed no findings in this regard. 


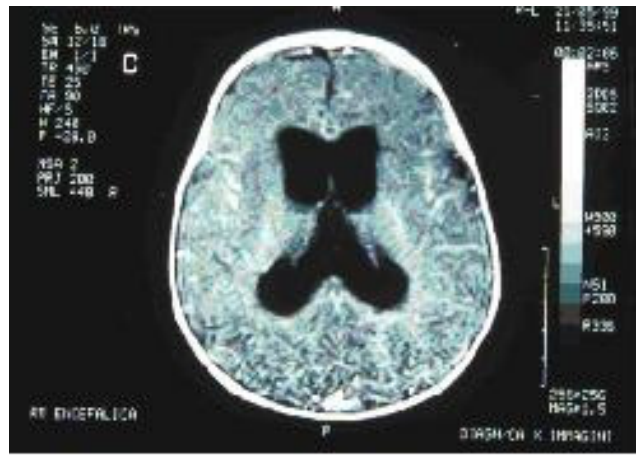

(a)

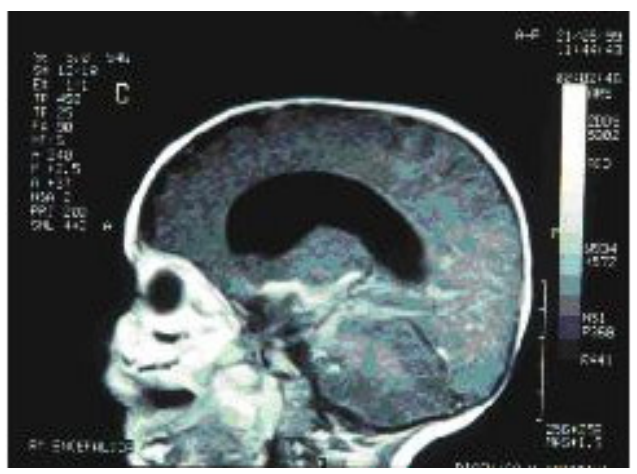

(b)

Fig. 1: Case 15. Transverse (a) and sagittal (b) cranial MRI showing severe tetraventricular hydrocephalus

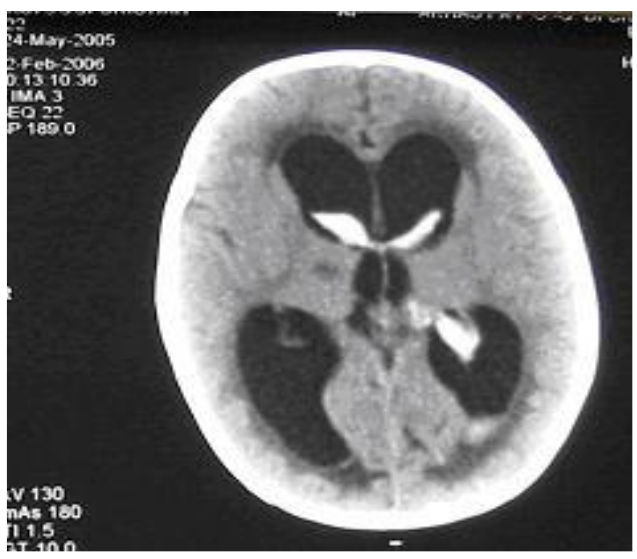

Fig. 2: Case 18. Transverse cranial CT showing severe tetraventricular hydrocephalus with intraventricular haemorrhage.

Various neurological sequelae (ventriculoperitoneal shunt, ptosis, motor-mental retardation, motor retardation) persisted in six children (33\%): half of these were in stage III of presentation.

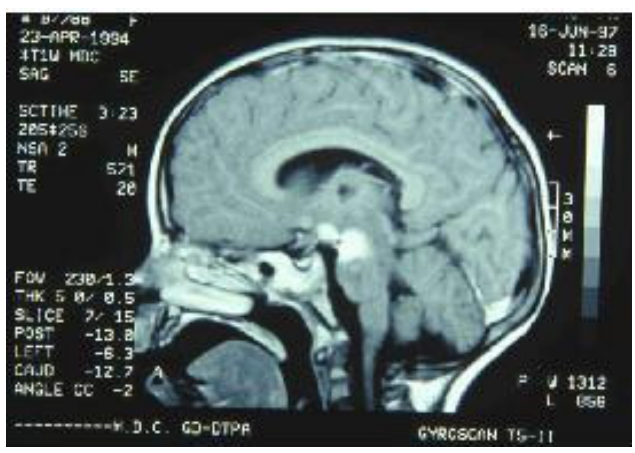

(a)

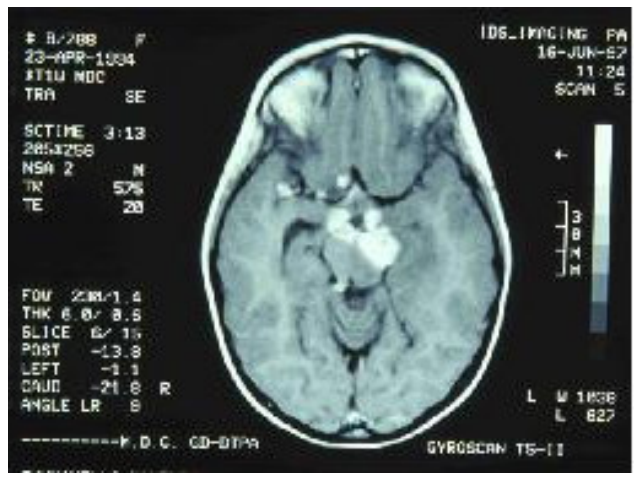

(b)

Fig. 3: Case 11 Sagittal (a) and transverse (b) cranial MRI showing a multiple mesencephalic nodular lesion

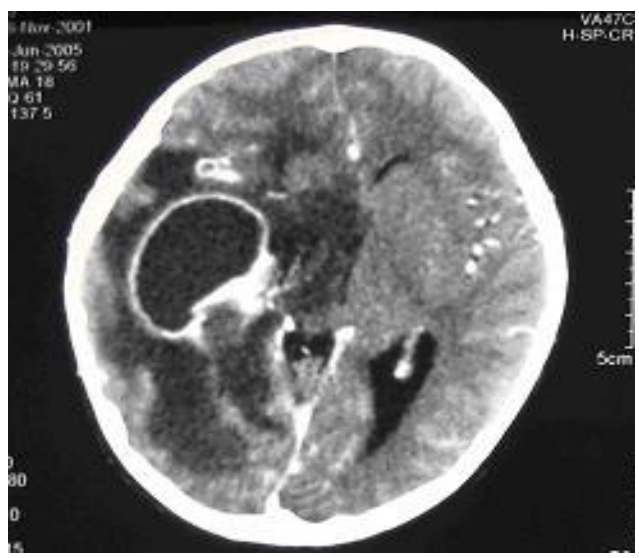

Fig. 4: Case 17 Transverse cranial CT showing lesion with mass effect, enhanced heterogeneity and surrounding edema in the right temporoparietal region

The analysis of neuroimaging findings (hydrocephalus, nodular lesions and ischemic areas) in relation to age on admission showed a statistically 
significant association between hydrocephalus and younger age $(\mathrm{p}=0.012)$.

The mean duration of hospitalization was 34.7 days (range: 12-75 days). The average follow-up was 36 months: 16 of the 18 patients (the 16 surviving patients???) were followed for ten years.

All the patients were treated with INH, RMP, PZA and SM or ETB, followed by 10 months of RMP and INH. We did not detect any clinical or microbiologic relapses during follow-up.

CNS-TB occurs either as a result of disseminated disease in infants or as a reactivation of disease in adolescents and adults. Disseminated disease typically occurs in immunocompromised patients and in young children, in whom a heavy burden of organisms develops in the face of a relatively immature immune response.

Despite great progress in laboratory research, many unresolved issues in paediatric tuberculosis diagnosis have still to be addressed. Cerebrospinal fluid (CSF) is usually negative and in children it may be difficult to obtain an adequate volume of fluid for culture. The sensitivity of smear microscopy and traditional culture is low and they give a late diagnosis. We obtained positive microbiological results for more than $50 \%$ of the children in our sample. Our hospital is a referral centre for paediatric infectious disease, therefore we were supported by a microbiologist with broad experience in tuberculosis diagnosis.

In the absence of microbiological data, CNS-TB diagnosis is achieved with a multi-approach system, i.e. with the analysis of CSF findings, the detection of both mycobacterial antigen and antibodies in CSF/serum, PCR assays and neuroimaging findings. These radiological technologies aid early diagnosis, suggesting a tuberculosis etiology based on major involvement of the basal encephalic region: hyperdensity in the basal cisterns seems to be typical of CNS-TB, although it is not a very sensitive finding (Naeisseria infection and subarachnoid hemorrhage are also reported as causes of hyperdensity in the basal cistern). All the neuroradiological investigations conducted on our patients revealed the thickening and intense enhancement of the meninges and, in accordance with the literature $^{[7]}$, a frequent basal cistern involvement.

A common characteristic of CNS infectious disease is the involvement of the ventricular system, especially in younger children under one year of age. Hydrocephalus per se is an unspecific lesion observed in bacterial meningitis, but the study of its features is related to a severe clinical picture ${ }^{[8]}$ and its incidence increases with the duration of illness. Both of our fatal cases had severe hydrocephalus and were in an advanced stage of clinical presentation. Hydrocephalus, lower age and stage of the disease are significant prognostic factors in CNS-TB. Half of the patients in our sample were $\leq 2$ years old and a lower age at presentation was associated with hydrocephalus. In a ten-month-old child, hydrocephalus was associated with extensive intraventricular haemorrhage (grade 3 ), as is frequently observed in premature infants. These findings are usually associated with a poor prognosis and sequelae.

The severity of hydrocephalus required the insertion of an external ventricular drainage system in six patients, which in three cases was converted into a permanent VP shunt in the absence of an improvement in their clinical condition. Palur suggested that early insertion of external drainage contributes to a better prognosis.

Clinical involvement of the cranial nerves has been reported in $17.4-70 \%$ of patients with CNS-TB ${ }^{[10]}$. Cranial nerve involvement without specific MR findings observed in our sample might be related to the ischemic changes of the cranial nerve nuclei or peripheral segments.

Ischemic lesions are prognostic determinants: changes in level of consciousness are more commonly observed in cases that develop infarctions and the prognosis is much worse for cases of stroke. These lesions result from thrombosis and occlusions secondary to panarteritis and can also occur adjacent to areas of severe meningeal and cisternal inflammation due to direct extension of the disease into the parenchyma.

We observed ischemic areas in two patients, both in clinical stage III, one had a fatal outcome and the other made a complete recovery. In patient $n^{\circ} 8$ a large embolus occluded the internal carotid lumen and the large extent of the ischemic area determined the death of the child.

None of our patients had tuberculomas. This might be due to the brief time of illness, considering the typical slow growth of this focal lesion. Although CT and conventional MRI findings have been described for the diagnosis of tuberculomas and tuberculous abscess, these are non-specific characteristics of the disease in a number of patients. Tuberculomas and tuberculous abscess constitute approximately 10 to $20 \%$ of all intracranial masses: a single enhancing CT documented lesion may occur in several CNS infectious and neoplastic diseases and is the most common radiological abnormality seen in patients with acuteonset seizures $^{[14]}$. 
The diagnostic accuracy of CT and MRI has greatly advanced the identification of CNS-TB. Although some findings are generally non-specific, modern imaging plays a key role in prompt diagnosis. A high index of suspicion in the appropriate clinical setting may lead to the application of specific therapeutic measures that can prevent unnecessary morbidity and mortality.

\section{REFERENCES}

1. Global tuberculosis control. WHO report 2000. WHO/CDS/TB/2000. 275:122-134. Geneva: WHO.

2. Moro, M.L., P. Malfait, G. Salamina and S. D'Amato, 1999. Tuberculosis in Italy: Available data and open questions. Epidemiol Prev., 23: 27-36.

3. Garg, R.K., 1999. Tuberculosis of the central nervous system. Postgrad Med. J., 75 (881): 133-40.

4. Peter, D. Corr, 2004. MBChB, eMedicine World Medical Library Tuberculosis, CNS,

5. Starke, J.R., 1998. Tuberculosis. In: Infectious diseases of children, Katz S.L., A.A. Gershon and P.J. Hotez, (Eds.), pp: 571-604, St. Louis: Mosby.

6. American Thoracic Society, 2000. Diagnostic standards and classification of tuberculosis in adults and children. Am. J. Resp. Crit. Med., 161: 1376-1395.

7. Dieli, F., G. Sireci, C. Di Sano, E. Champagne, J.J. Fournie and A. Salerno, 1999. Predominance of Vgamma9/Vdelta2 T lymphocytes in cerebrospinal fluid of children with tuberculous meningitis: Reversal after chemotherapy. Mol. Med., 5: 301-12.7.
8. Andronikou, S., B. Smith, M. Hatherhill, H. Douis and J. Wilmshurst, 2004. Definitive neuroradiological diagnostic features of tuberculous meningitis in children. Pediatr Radiol., 34 (11): 876-85.

9. Shcoeman, J.F., J.A. Laubscher and P.R. Donald, 2000. Serial lumbar CSF pressure measurements and cranial computed tomographic findings in childhood tubercular meningitis. Child. Nerv. Syst., 16: 203-208.

10. Uysal, G., G. Köse and B. Güven Diren, 2001. Magnetic Resonance imaging in diagnosis of childhood central nervous system tuberculosis. Infection, 29: 148-153.

11. CDC. Case definitions for infectious conditions under public health surveillance 1997, MWR, 46 (No. RR-10): 40-41.

12. Kennedy, D.H. and R.J. Fallon, 1979. Tuberculous meningitis. JAMA., 241: 264-268.

13. American Thoracic Society. Treatment of tuberculosis and tuberculosis infection in adults and children, 1994. Am. J. Resp. Crit. Med., 149: 1359-74.

14. Ravindra Kumar Garg, 2002. Single enhancing computerized tomography-detected lesion in immunocompetent patients. Neurosurg Focus, $12(6)$. 